\title{
Asymptomatic cytomegalovirus infection is associated with increased risk of new-onset diabetes mellitus and impaired insulin release after renal transplantation
}

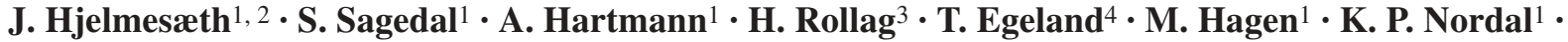 \\ T. Jenssen ${ }^{1}$ \\ ${ }^{1}$ Department of Medicine, Section of Nephrology, Rikshospitalet University Hospital, Oslo, Norway \\ 2 Department of Medicine, Vestfold Hospital HF, Tønsberg, Norway \\ ${ }^{3}$ Institute of Medical Microbiology, Rikshospitalet University Hospital, Oslo, Norway \\ ${ }^{4}$ Biostatistics, Rikshospitalet University Hospital, Oslo, Norway
}

\begin{abstract}
Aims/hypothesis. The human cytomegalovirus (CMV) may increase the risk of diabetes mellitus, but the literature is scarce. The present study was designed to test the hypothesis that asymptomatic CMV infection is associated with increased risk of new-onset diabetes after renal transplantation, and to assess the impact of asymptomatic CMV infection on OGTT-derived estimates of insulin release and insulin action.

Methods. A total of 160 consecutive non-diabetic renal transplant recipients on cyclosporine (Sandimmun Neoral)-based immunosuppression were closely monitored for CMV infection during the first 3 months after transplantation. All patients underwent a 75-g OGTT at 10 weeks. Excluded from the analyses were 36 patients with symptomatic CMV infection (disease).
\end{abstract}

Results. The incidence of new-onset diabetes was $6 \%$ in a control group of recipients without CMV infection $(4 / 63)$ and $26 \%$ in the group with asymptomatic CMV infection (16/61). Asymptomatic CMV infection was associated with a significantly increased risk of new-onset diabetes (adjusted odds ratio: 4.00; 95\% CI: 1.19 to $13.43, p=0.025)$. The group of patients with CMV infection had a significantly lower median insulin release than controls.

Conclusions/interpretation. Our findings support the hypothesis that asymptomatic CMV infection is associated with increased risk of new-onset post-transplant diabetes mellitus, and suggest that impaired insulin release may involve one pathogenetic mechanism.

Keywords Cyclosporine - Cytomegalovirus infections · Diabetes mellitus · Etiology · Glucocorticoids · Glucose tolerance test $\cdot$ Insulin $\cdot$ Kidney transplantation

\section{Introduction}

The hypothesis that virus infections may provoke Type 1 diabetes in genetically predisposed individuals is generally accepted [1]. Both direct viral infection of

Received: 1 October 2003 / Accepted: 5 January 2004

Published online: 27 August 2004

C) Springer-Verlag 2004

\section{J. Hjelmesæth (ه)}

Department of Medicine, Vestfold Hospital HF, Boks 2168,

3103 Tønsberg, Norway

E-mail: joran@online.no

Tel.: +47-33-342000, Fax: +47-33-343938

Abbreviations: Anti-GAD, antibodies to GAD · Anti-HCV, antibodies to hepatitis $\mathrm{C}$ virus - CMV, cytomegalovirus . $\mathrm{HCV}$, hepatitis $\mathrm{C}$ virus - PTDM, post-transplantation diabetes mellitus the beta cell and immune-mediated responses have been proposed as pathogenetic mechanisms. Viruses reported to be associated with Type 1 diabetes are coxsackie B virus, rubella virus, mumps virus, EpsteinBarr virus, varicella zoster virus and cytomegalovirus (CMV) [1].

Interestingly, recently published studies have indicated that virus infections may also increase the risk of other types of diabetes. From the Third National Health and Nutrition Examination Survey in the USA it was reported that persons with hepatitis $\mathrm{C}$ virus (HCV) infection, as defined by a positive antibody test to $\mathrm{HCV}$, were more than three times more likely to have Type 2 diabetes mellitus than those without infection [2]. In addition, HCV infection has been associated with new-onset post-transplantation diabetes (PTDM) in renal transplant recipients treated with tacrolimus [3]. Moreover, patients infected with hu- 


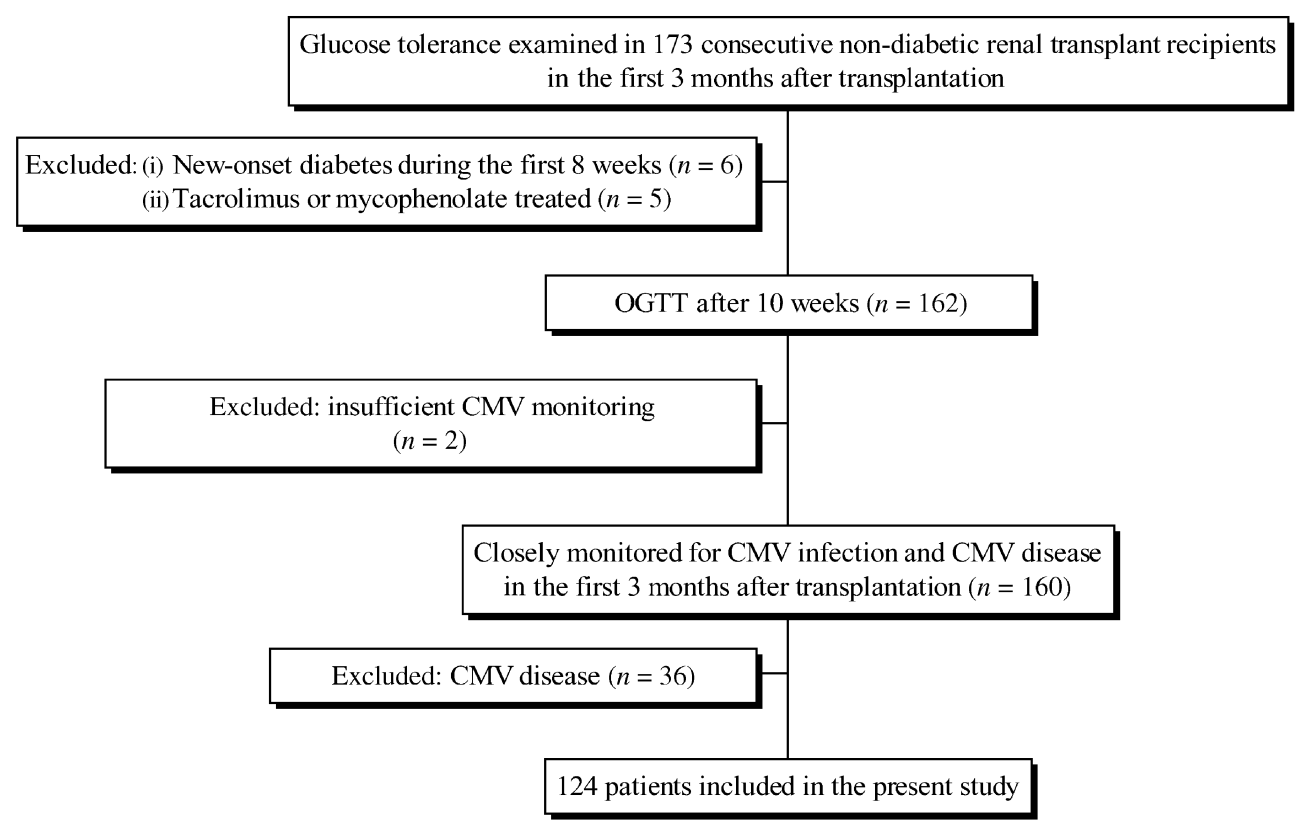

Fig. 1. Study design

man immunodeficiency virus may be prone to glucose intolerance and insulin resistance [4].

A strong correlation between the presence of the human CMV genome in lymphocytes and serum islet cell autoantibodies in newly diagnosed Type 1 diabetic patients has previously been reported [5]. Moreover, it has been argued that human CMV may play an important role in the pathogenesis of Type 1 diabetes through $\mathrm{T}$ cell crossreactivity with the beta cell autoantigen GAD65 [5, 6]. CMV can also infect pancreatic islet cells in vivo [7] and has been detected in islet cells from both Type 1 [5] and Type 2 [8] diabetic patients.

Even though it has been speculated that CMV increases the risk of diabetes, the literature is scarce, probably due to the low incidence of active viral infection and Type 1 diabetes in the general population. On the other hand, both CMV infection and new-onset diabetes are frequently observed in renal transplant recipients $[9,10]$. Therefore, this group of patients is of particular interest for the study of a potential relationship between CMV and diabetes. Moreover, the results of a recently published study indicate that impaired beta cell antiviral defence may increase the risk of developing diabetes after infection with CMV [11].

Glucose intolerance is a well-known side effect of immunosuppressive therapy. Calcineurin inhibitors probably impair insulin release, and steroids may have negative effects on insulin action and secretion [12, $13,14,15,16]$. In this respect, PTDM shares features with both Type 1 and Type 2 diabetes.

We have previously argued that patients with ganciclovir-treated symptomatic CMV disease may have increased risk of developing new-onset PTDM [9], and that CMV disease may be associated with insulin resistance [15] and beta cell dysfunction [17] after renal transplantation. However, a heterogeneous control group consisting of subjects with asymptomatic CMV infection as well as persons without CMV infection may have flawed the results. Moreover, the effects of asymptomatic CMV infection on glucose tolerance, insulin release and insulin action have not been addressed previously.

Thus, the objectives of the present study were as follows: (i) to examine whether renal transplant recipients with asymptomatic CMV infection are at increased risk of developing new-onset PTDM as compared with transplanted subjects without infection (controls); and (ii) to assess whether asymptomatic CMV infection has a negative impact on insulin release, insulin action or both.

\section{Subjects and methods}

Study design. The design of this study is illustrated in Figure 1. Glucose intolerance was prospectively examined in 173 consecutive non-diabetic renal transplant recipients at Rikshospitalet University Hospital during the first 3 months after transplantation over a period of 16 months from 1995 to 1996 [9]. To avoid the potentially confounding effect of various immunosuppressive protocols, patients treated with tacrolimus $(n=4)$ or mycophenolate $(n=1)$ were excluded. Another six patients with manifest new-onset diabetes at an early stage ( $0-8$ weeks) did not undergo an OGTT and were excluded because insulin secretion and insulin action could not be assessed.

The remaining 162 patients underwent a 75-g OGTT 10 weeks $(70 \pm 6$ days, mean \pm SD) after transplantation $[9,15]$. The majority of these patients $(n=160)$ were also closely monitored for CMV infection and CMV disease during the first 3 months after transplantation [10]. Taking into account the objectives of the present study, the 36 patients with CMV disease were excluded, leaving a total of 124 recipients for inclusion in the present analysis. All patients gave informed consent 
Table 1. Demography, rejections, immunosuppressive therapy and renal function 10 weeks after renal transplantation

\begin{tabular}{|c|c|c|c|}
\hline & $\begin{array}{l}\text { Asymptomatic } \\
\text { CMV infection }\end{array}$ & No CMV infection & $p$ value \\
\hline Patients ${ }^{a}$ & $61(49 \%)$ & $63(51 \%)$ & \\
\hline Patients with a family history of diabetes ${ }^{a}$ & $14(25 \%)$ & $12(20 \%)$ & 0.522 \\
\hline Patients with one or more rejections ${ }^{\mathrm{a}}$ & $42(69 \%)$ & $25(40 \%)$ & 0.001 \\
\hline Age $(\text { years })^{b}$ & $53 \pm 15$ & $43 \pm 15$ & $<0.001$ \\
\hline Creatinine $(\mu \mathrm{mol} / \mathrm{l})^{\mathrm{c}}$ & $138(85-284)$ & $133(64-224)$ & 0.484 \\
\hline Cyclosporine A through concentration $(\mu \mathrm{g} / \mathrm{l})^{\mathrm{c}}$ & $250(110-528)$ & $230(123-400)$ & 0.058 \\
\hline
\end{tabular}

${ }^{\text {a }}$ Data are given as $n(\%) ;{ }^{\mathrm{b}}$ data are given as means $\pm \mathrm{SD} ;{ }^{\mathrm{c}}$ data are given as medians (range). Unpaired $t$ test, Mann-Whitney test or Pearson chi square used as appropriate

to participate, and the study protocol was approved by the Regional Committee for Medical Research Ethics (Health Region South).

The immunosuppressive regimen consisted of oral prednisolone and cyclosporine A (Sandimmun Neoral; Sandoz Pharma/Novartis) (all patients) and azathioprine $(n=139)$. Details on the immunosuppressive protocol have been described previously [9].

In the present study CMV infection was monitored by measuring CMV pp65 lower matrix protein in EDTA blood samples. Patients were screened at least every second week during the study. The assay for CMV pp65 antigen was performed as previously described [18]. The result was given as the number of CMV pp65 antigen-positive cells per 100,000 leucocytes. Patients with CMV pp65 antigenaemia of 1 or more per 100,000 leucocytes were classified as having asymptomatic CMV infection or CMV disease depending on the absence or presence of clinical symptoms or signs. The criteria for CMV infection and disease implemented in the present study are consistent with recently suggested definitions [19].

The presence of antibodies to HCV (anti-HCV) was assessed by a third-generation enzyme immunoassay (Axzym HCV 3.0; Abbott Diagnostics, Chicago, Ill., USA). Reactive samples were further tested on an anti-HCV immunoblot (RIBA HCV 3.0; Chiron, Emeryville, Calif., USA). Sera from all donors were examined and found negative for anti-HCV. The recipients were screened for anti-HCV two weeks (12 \pm 7 days) before the OGTT.

Antibodies against the human $65-\mathrm{M}_{\mathrm{r}}$ isoform of GAD (antiGAD65) were assayed in serum samples by immune precipitation as previously described [20].

Oral glucose tolerance test. After an overnight fast, a 75-g OGTT was performed, and serum glucose and insulin were measured at 0, 1 and 2 hours as previously described [15]. The patients were categorised according to the diagnostic criteria for glucose tolerance suggested by the Expert Committee [21]. All patients completed a questionnaire with respect to diabetes among first-degree relatives.

Insulin sensitivity and secretion indices. We have recently validated six OGTT-derived insulin sensitivity indices (ISIs) against an insulin sensitivity index derived from the euglycaemic-hyperinsulinaemic glucose clamp in renal transplant recipients [15]. The $\mathrm{ISI}_{\mathrm{TX}}$, which is a slight modification of an ISI proposed by Stumvoll and co-workers [22], correlated best with the clamp results and was used in estimating insulin $\mathrm{s}$ ensitivity in the present study $\left(\mathrm{ISI}_{\mathrm{TX}}=0.208-0.0032 \times \mathrm{BMI}-\right.$
$0.0000645 \times$ Ins $_{120}-0.00375 \times$ Gluc $_{120} ;$ Ins $_{120}$ and Gluc $_{120}$ are OGTT serum insulin and glucose values after $120 \mathrm{~min}$ ).

Insulin release was estimated using three equations, which have been shown to correlate well with results of hyperglycaemic clamp studies [22, 23, 24]. The AUC insulin and the AUC glucose during the OGTT were implemented in the insulin release index: $\operatorname{Secr}_{\mathrm{AUC}}=\mathrm{AUC}_{\mathrm{Ins}} / \mathrm{AUC}_{\mathrm{Gluc}}$. The first- and second-phase insulin releases were estimated implementing insulin values at 0 and $60 \mathrm{~min}$, and glucose at $60 \mathrm{~min}$ : Secr $_{1 \text { st phase }}=1194+4.724 \times \mathrm{Ins}_{0}-117.0 \times \mathrm{Gluc}_{60}+1.414 \times \mathrm{Ins}_{60}$ and Secr $_{\text {2nd phase }}=295+0.349 \times \mathrm{Ins}_{60}-25.72 \times$ Gluc $_{60}+1.107 \times \mathrm{Ins}_{0}$.

Statistical analysis. Unpaired $t$ test, Mann-Whitney test, Pearson chi square, Fisher's Exact Test and logistic or linear multiple regression were used as appropriate. Two-tailed $p$ values of below 0.05 were considered significant. The analysis was implemented using SPSS version 10.0 statistical software (SPSS, Chicago, Ill., USA).

From previous publications it was known that the incidence of new-onset PTDM was about 17\% [15], and that approximately $35 \%$ of the patients had asymptomatic CMV infection [10]. Taking this into account, the present study had a power of more than $80 \%(\alpha=0.05)$ to detect a five-fold increased risk of PTDM in patients with asymptomatic CMV infection, as compared with controls without CMV infection.

\section{Results}

Patient demographics according to CMV category are shown in Table 1. A total of 61 patients (49\%) had asymptomatic CMV infection compared with 63 subjects $(51 \%)$ with no signs of CMV infection (control group). CMV pp65 antigenaemia was registered after a median (range) of 33 days (12-74). No patients tested positive for HCV. All recipients with PTDM were anti-GAD-negative. Only one patient, who had CMV infection in the presence of normoglycaemia, tested positive for anti-GAD.

The patients with CMV infection were significantly older $(p<0.001)$, and had a higher frequency of rejection episodes $(p=0.001)$ than recipients in the control group. Statistically non-significant trends towards lower median BMI and cyclosporine through concen- 
tration in the control group were observed. The median daily dose of prednisolone did not differ significantly between the groups $(p=0.413)$.

CMV infection and glucose tolerance. Relative proportions of patients with varying degrees of glucose tolerance according to CMV status are shown in Figure 2. A significantly higher proportion of patients with CMV infection (26\%) developed PTDM compared with controls $(6 \% ; p=0.003$; Table 2). The median 2-h blood glucose was also significantly higher in patients with CMV infection compared with control patients (Table 2).

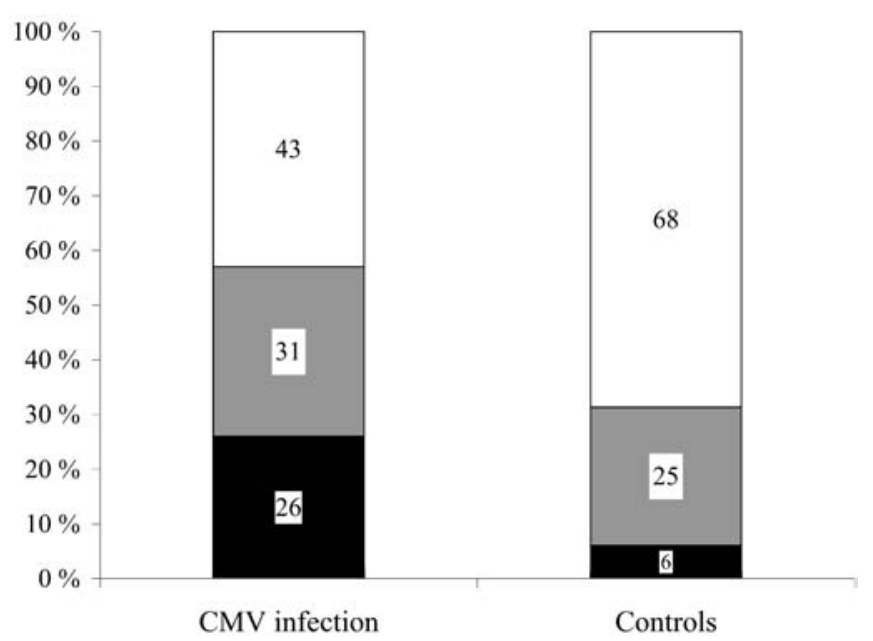

Fig. 2. The relative proportion of patients with varying degrees of glucose tolerance in patients with asymptomatic CMV infection $(n=61)$ and patients without CMV infection (controls, $n=63)$. White, NGT; grey, IGT/IFG; black, PTDM
Table 3 shows that patients with asymptomatic CMV infection had a five-fold increased risk of PTDM (odds ratio: 5.24) in the univariate logistic regression analysis. To adjust for potentially confounding factors, documented risk factors for PTDM, such as age, family history of diabetes, BMI, daily prednisolone dose and rejection (yes/no), were included in addition to CMV infection as the independent variable, and PTDM (yes/no) as the dependent variable in a multiple forward stepwise logistic regression analysis. CMV infection (odds ratio: $4.00 ; 95 \% \mathrm{CI}: 1.19$ to 13.43, $p=0.025$ ) and age remained significantly associated with increased risk of PTDM after multivariate analysis (Table 3 ).

Insulin release and insulin action according to CMV category. Table 4 shows that estimated median insulin secretion was significantly lower in patients with asymptomatic CMV infection than in controls (all insulin secretion indices, $p<0.001$ ), whereas the estimated median insulin sensitivity did not differ significantly. In addition, to adjust for potentially confounding variables known to affect insulin release, multiple linear regression analyses were performed using the lntransformed insulin release indices as dependent variables, and CMV infection, insulin sensitivity and age as independent variables. After correction for age and insulin sensitivity, asymptomatic CMV infection was still associated with lower insulin secretion $\left(\operatorname{Secr}_{\mathrm{AUC}}\right.$, $p=0.003 ;$ Secr $_{1 \text { st phase, }} p=0.026 ; \operatorname{Secr}_{2 \text { nd phase, }} p=0.011$ ).

To correct for the negative effect of hyperglycaemia on insulin release, the subgroup of patients

Table 2. Frequency of PTDM and median serum glucose concentrations according to CMV category

\begin{tabular}{llll}
\hline & $\begin{array}{l}\text { Asymptomatic CMV infection } \\
(n=61)\end{array}$ & $\begin{array}{l}\text { No CMV infection } \\
(n=63)\end{array}$ & $p$ value \\
\hline Patients with PTDM & $16(26.2 \%)$ & $4(6.3 \%)$ & $0.003(0.003)$ \\
Fasting glucose (mmol/1) & $5.4(4.4-13.3)$ & $5.4(3.5-7.3)$ & 0.798 \\
2-h glucose (mmol/l) & $7.8(4.0-21.5)$ & $6.9(4.0-13.1)$ & 0.008 \\
\hline
\end{tabular}

Data are given as $n$ (\% of total) or medians (range). Pearson chi square (Fisher's exact test) or Mann-Whitney test used as appropriate

Table 3. Asymptomatic CMV infection as an independent risk factor for new-onset post-transplantation diabetes mellitus

\begin{tabular}{|c|c|c|c|c|c|c|}
\hline & $p$ value & Odds ratio & $95 \% \mathrm{CI}$ & $p$ value & Odds ratio & $95 \% \mathrm{CI}$ \\
\hline Age & 0.004 & 1.05 & $1.02-1.09$ & 0.047 & 1.04 & $1.00-1.08$ \\
\hline Daily dose of prednisolone & 0.183 & 1.05 & $0.98-1.13$ & NA & & \\
\hline Family history of diabetes & 0.349 & 1.67 & $0.57-4.90$ & NA & & \\
\hline
\end{tabular}

Shown are the results of the univariate regression (unadjusted) and the multiple forward stepwise logistic regression (adjusted). NA, not applicable (i.e. not in the equation) 
Table 4. Estimated insulin secretion and insulin sensitivity according to CMV status, including all patients

\begin{tabular}{lccr}
\hline & $\begin{array}{l}\text { Asymptomatic CMV infection } \\
(n=61)\end{array}$ & $\begin{array}{l}\text { No CMV infection } \\
(n=63)\end{array}$ & $p$ value \\
\hline Insulin sensitivity, ISI $_{\text {TX }}\left(\times 10^{-2}\right)$ & $7.6(0.6-12.0)$ & $7.8(0.2-12.1)$ & 0.511 \\
First-phase insulin secretion & $1191(-262$ to 6232) & $1505(80-4110)$ & $<0.001$ \\
Second-phase insulin secretion & $309(-7$ to 1557$)$ & $385(66-1023)$ & $<0.001$ \\
AUC for insulin secretion & $41(9-323)$ & $53(16-190)$ & $<0.001$ \\
\hline
\end{tabular}

Data are given as medians (range). Mann-Whitney test used

Table 5. Estimated median insulin secretion and insulin sensitivity according to CMV status, only including patients with NGT

\begin{tabular}{lccc}
\hline & $\begin{array}{l}\text { Asymptomatic CMV infection } \\
(n=26)\end{array}$ & $\begin{array}{l}\text { No CMV infection } \\
(n=43)\end{array}$ & $p$ value \\
\hline Insulin sensitivity, SSI $_{\mathrm{TX}}\left(\times 10^{-2}\right)$ & $9.1(5.0-12.0)$ & $8.9(2.9-12.1)$ & 0.720 \\
First-phase insulin secretion & $1346(990-6231)$ & $1629(593-4110)$ & 0.003 \\
Second-phase insulin secretion & $367(265-1557)$ & $418(170-1023)$ & 0.005 \\
AUC for insulin secretion & $45(33-323)$ & $58(16-190)$ & 0.006 \\
\hline
\end{tabular}

Data are given as medians (range). Mann-Whitney test used

with NGT was included in a second assessment of insulin release and insulin action (Table 5). The results were essentially unaltered, and the estimated median insulin secretion was significantly lower in patients with asymptomatic CMV infection compared with controls, also in this subgroup. Moreover, no significant differences in estimated insulin sensitivity between the different groups were found. Inclusion of age in the model did not alter the results significantly.

\section{Discussion}

We have previously reported that symptomatic CMV disease may be a risk factor for the development of new-onset diabetes mellitus after renal transplantation [9]. An important limitation of our previous study was that the control group included both patients with asymptomatic CMV infection and patients without CMV infection.

A major and novel finding of the present prospective cohort study is that renal transplant recipients with asymptomatic CMV infection, too, had a significantly increased risk of new-onset diabetes mellitus compared with a control group of patients without CMV infection.

Clearly, the present study does not prove a causal relationship between CMV infection and diabetes, but it does add some support to the hypothesis that CMV may provoke the development of diabetes in predisposed individuals. CMV infection was detected prior to the diagnosis of diabetes, and a thorough surveillance of the incidence of CMV infection was performed, including blood samples drawn at least every second week for 3 months. Although the median time to the registration of CMV infection was 5 weeks, whereas the OGTT was performed after 10 weeks, we cannot completely rule out the possibility that some patients may have become diabetic before they were infected with CMV.

Moreover, CMV infection represents only one of a number of potentially modifiable risk factors for newonset diabetes such as immunosuppressive drugs, rejections and body weight. Nevertheless, the results of the present study indicate that CMV infection is an important and independent risk factor for PTDM irrespective of rejection episodes and immunosuppressive therapy.

Our results are consistent with some previous epidemiological studies and case reports $[1,5]$, but contrast with the negative results of a large long-term study of maternal and congenital CMV infection in Sweden [25]. However, in view of the very low incidence of Type 1 diabetes in the latter study, the apparent negative outcome may represent a statistical type II error. The authors actually reported a numerical seven-fold higher incidence of Type 1 diabetes in congenitally infected infants than in non-infected children $(1.4 \%$ [1/76] vs $0.2 \%$ [38/19.483], $p=0.14)$. Given these incidence figures, the power to detect a sevenfold higher risk of diabetes in the CMV-infected children was less than $35 \%(\alpha=5 \%)$.

The present study strongly indicates that impaired insulin release explains the association between asymptomatic CMV infection and new-onset diabetes after transplantation. Patients with asymptomatic CMV infection had significantly lower OGTT-derived median insulin secretion than controls, even after adjustments for confounding factors. Although we did not address cellular or molecular pathogenetic mecha- 
nisms, we found that no individual with new-onset diabetes tested positive for anti-GAD. This finding supports the notion that the pathogenesis of PTDM may be different from that of Type 1 diabetes mellitus. On the other hand, the results of a recently published study on animals indicate that impaired beta cell antiviral defence may enhance susceptibility to diabetes triggered by CMV [11]. In addition, renal transplant recipients with CMV infection may have increased activation of the TNF system [26], which has been shown to be harmful to beta cells in in vitro cultures [1]. In view of these findings, one may speculate that more direct or indirect effects of CMV infection on the beta cell may be involved in the pathogenesis of new-onset PTDM.

No significant differences in insulin action were documented in the present study. This is in contrast with our previous findings, which suggested that patients with CMV disease were insulin resistant [15]. However, our previous results were probably flawed by the fact that the "control" group consisted of patients both with and without CMV infection. Nevertheless, it is conceivable that CMV infection may induce insulin resistance through release of cytokines and TNF- $\alpha$ [27], and the present study had insufficient power to detect any potential small differences in insulin sensitivity.

It is generally accepted that some patients with early-diagnosed PTDM may improve their glucose tolerance during later tapering of immunosuppressive treatment. In a 1-year follow-up study we reported that about half of the recipients with early-diagnosed PTDM improved to NGT or IGT [28]. A post hoc analysis of the follow-up data revealed that six of the 14 PTDM patients included in the present study remained diabetic. Interestingly, all four patients with PTDM and no signs of CMV infection at baseline improved to NGT after 1 year.

Implications. The results of the present study may have implications for the diagnosis, prophylaxis and treatment of asymptomatic CMV infection after renal transplantation. Prophylactic treatment with oral valacyclovir during the first 3 months after transplantation significantly reduced the incidence of CMV viraemia and disease in a large placebo-controlled study including about 600 renal transplant recipients [29]. A strategy of pre-emptive treatment of patients with asymptomatic CMV infection with intravenous [30] or oral [31] ganciclovir has also shown to be effective in reducing the incidence of CMV disease. However, the effects of CMV prophylaxis and pre-emptive antiCMV therapy on the incidence of new-onset PTDM remain to be assessed in future controlled studies.

Conclusions. We conclude that our findings support the hypothesis that asymptomatic CMV infection increases the risk of new-onset diabetes mellitus after renal transplantation, and suggest that impaired insulin release may involve one pathogenetic mechanism.

Acknowledgements. This study was supported by the Norwegian Foundation for Health and Rehabilitation, the Research Council of Norway and Vestfold Hospital HF. The authors wish to thank the bioengineers Jean Stenstrøm, Jannicke Narverud, Kirsten Lund and Els Breistein from the Laboratory for Renal Physiology for investing a lot of effort into the laboratory work.

\section{References}

1. Jaeckel E, Mann M, von Herrath M (2002) Viruses and diabetes. Ann NY Acad Sci 958:7-25

2. Mehta SH, Brancati FL, Sulkowski MS, Strathdee SA, Szklo M, Thomas DL (2000) Prevalence of type 2 diabetes mellitus among persons with hepatitis $\mathrm{C}$ virus infection in the United States. Ann Intern Med 133:592-599

3. Bloom RD, Rao V, Weng F, Grossman RA, Cohen D, Mange KC (2002) Association of hepatitis C with posttransplant diabetes in renal transplant patients on tacrolimus. J Am Soc Nephrol 13:1374-1380

4. Dubé MP (2000) Disorders of glucose metabolism in patients infected with human immunodeficiency virus. Clin Infect Dis 31:1467-1475

5. Pak CY, Eun HM, McArthur RG, Yoon JW (1988) Association of cytomegalovirus infection with autoimmune type 1 diabetes. Lancet 2:1-4

6. Hiemstra HS, Schloot NC, van Veelen PA et al. (2001) Cytomegalovirus in autoimmunity: $\mathrm{T}$ cell crossreactivity to viral antigen and autoantigen glutamic acid decarboxylase. Proc Natl Acad Sci USA 98:3988-3991

7. Jenson AB, Rosenberg HS, Notkins AL (1980) Pancreatic islet-cell damage in children with fatal viral infections. Lancet 2:354-358

8. Lohr JM, Oldstone MB (1990) Detection of cytomegalovirus nucleic acid sequences in pancreas in type 2 diabetes. Lancet 336:644-648

9. Hjelmesæth J, Hartmann A, Kofstad J et al. (1997) Glucose intolerance after renal transplantation depends upon prednisolone dose and recipient age. Transplantation 64:979983

10. Sagedal S, Nordal KP, Hartmann A et al. (2000) A prospective study of the natural course of cytomegalovirus infection and disease in renal allograft recipients. Transplantation 70:1166-1174

11. Flodstrom M, Tsai D, Fine C, Maday A, Sarvetnick N (2003) Diabetogenic potential of human pathogens uncovered in experimentally permissive $\beta$-cells. Diabetes 52:2025-2034

12. Ekstrand AV, Eriksson JG, Grönhagen-Riska C, Ahonen PJ, Groop LC (1992) Insulin resistance and insulin deficiency in the pathogenesis of posttransplant diabetes in man. Transplantation 53:563-569

13. Midtvedt K, Hartmann A, Hjelmesæth J, Lund K, Bjerkely $\mathrm{BL}$ (1998) Insulin resistance is a common denominator of post-transplant diabetes mellitus and impaired glucose tolerance in renal transplant recipients. Nephrol Dial Transplant 13:427-431

14. Nam JH, Mun JI, Kim SI et al. (2001) $\beta$-cell dysfunction rather than insulin resistance is the main contributing factor for the development of postrenal transplantation diabetes mellitus. Transplantation 71:1417-1423 
15. Hjelmesæth J, Midtvedt K, Jenssen T, Hartmann A (2001) Insulin resistance after renal transplantation: impact of immunosuppressive and antihypertensive therapy. Diabetes Care 24:2121-2126

16. Lambilotte C, Gilon P, Henquin JC (1997) Direct glucocorticoid inhibition of insulin secretion. An in vitro study of dexamethasone effects in mouse islets. J Clin Invest 99:414-423

17. Hjelmesæth J, Jenssen T, Hagen M, Egeland T, Hartmann A (2003) Determinants of insulin secretion after renal transplantation. Metabolism 52:573-578

18. Rollag H, Sagedal S, Holter E, Degre M, Ariansen S, Nordal KP (1998) Diagnosis of cytomegalovirus infection in kidney transplant recipients by a quantitative RNA-DNA hybrid capture assay for cytomegalovirus DNA in leukocytes. Eur J Clin Microbiol Infect Dis 17:124-127

19. Ljungman P, Griffiths P, Paya C (2002) Definition of cytomegalovirus infection and disease in transplant recipients. Clin Infect Dis 34:1094-1097

20. Birkeland KI, Kilhovd B, Thorsby P et al. (2003) Heterogeneity of non-insulin-dependent diabetes expressed as variability in insulin sensitivity, $\beta$-cell function and cardiovascular risk profile. Diabet Med 20:37-45

21. The Expert Committee on the Diagnosis and Classification of Diabetes Mellitus (2000) Report of the Expert Committee on the Diagnosis and Classification of Diabetes Mellitus. Diabetes Care 23 [Suppl 1]:S4-S19

22. Stumvoll M, Mitrakou A, Pimenta W et al. (2000) Use of the oral glucose tolerance test to assess insulin release and insulin sensitivity. Diabetes Care 23:295-301

23. Stumvoll M, Van Haeften T, Fritsche A, Gerich J (2001) Oral glucose tolerance test indexes for insulin sensitivity and secretion based on various availabilities of sampling times. Diabetes Care 24:796-797
24. Stumvoll M, Mitrakou A, Pimenta W et al. (2000) Assessment of insulin secretion from the oral glucose tolerance test in white patients with type 2 diabetes. Diabetes Care 23:1440-1441

25. Ivarsson SA, Lindberg B, Nilsson KO, Ahlfors K, Svanberg L (1993) The prevalence of type 1 diabetes mellitus at follow-up of Swedish infants congenitally infected with cytomegalovirus. Diabet Med 10:521-523

26. Nordøy I, Müller F, Nordal KP et al. (2000) The role of the tumor necrosis factor system and interleukin-10 during cytomegalovirus infection in renal transplant recipients. J Infect Dis 181:51-57

27. Moller DE (2000) Potential role of TNF- $\alpha$ in the pathogenesis of insulin resistance and type 2 diabetes. Trends Endocrinol Metab 11:212-217

28. Hjelmesæth J, Hartmann A, Kofstad J, Egeland T, Stenstrøm J, Fauchald P (2001) Tapering off prednisolone and cyclosporin the first year after transplantation: the effect on glucose tolerance. Nephrol Dial Transplant $16: 829-835$

29. Lowance D, Neumayer HH, Legendre CM et al. (1999) Valacyclovir for the prevention of cytomegalovirus disease after renal transplantation. $N$ Engl J Med 340:14621470

30. Künzle N, Petignat C, Francioli P et al. (2000) Preemptive treatment approach to cytomegalovirus (CMV) infection in solid organ transplant patients: relationship between compliance with the guidelines and prevention of CMV morbidity. Transpl Infect Dis 2:118-126

31. Sagedal S, Nordal KP, Hartmann A et al. (2003) Preemptive therapy of CMVpp65 antigen positive renal transplant recipients with oral ganciclovir: a randomized, comparative study. Nephrol Dial Transplant 18:18991908 